\title{
New insight on diversity of the Nikita Botanical Gardens plant collections from advanced NGS technology
}

\author{
Irina Mitrofanova \\ NBG-NSC RAS, Yalta, Russia \\ irimitrofanova@yandex.ru \\ Svetlana Chelombit \\ NBG-NSC RAS, Yalta, Russia \\ chelombit@inbox.ru
}

\author{
Olga Krivenko \\ NBG-NSC RAS, Yalta, Russia \\ olkrivenko@gmail.com \\ Valentina Brailko \\ NBG-NSC RAS, Yalta, Russia \\ labgennbs@yandex.ru
}

\author{
Olga Kuleshova \\ NBG-NSC RAS, Yalta, Russia \\ v_olgo4ka@inbox.ru \\ Olga Mitrofanova \\ NBG-NSC RAS, Yalta, Russia \\ invitro_plant@yandex.ru
}

\begin{abstract}
New scientific program of genomic screening of the Nikita Botanical Gardens plant collections targeted at comprehensive description of their molecular diversity in close connection with morphological, physiological and others phenotype features has started. Strategic goal is to develop and implement advanced molecular approaches for plant biotechnology, breeding, propagation, and conservation routine works. Two whole-genome sequencing projects to produce a high quality assembly of the reference genomes of the common fig Ficus carica L. (Moraceae) and Crimean endemic species Heracleum ligusticifolium M. Bieb. (Apiaceae) are in progress. Several hybrid assembly approaches using combined data sets with long and short reads obtained by Oxford Nanopore MinION and Illumina NextSeq550 instruments are tested to create accurate and well-resolved reference genome. The first versions of the reference genomes of $F$. carica cultivar 'Sabrutsiya Rozovaya' and $\boldsymbol{H}$. ligusticifolium were made, and their annotations are performing. Subsequent improvement and development of the primary genome assemblies up to "pangenome" rank will be carried out using advanced bioinformation approaches and computational tools by assimilation of new sequenced genomes and transcriptomes of the model species taken from the NBS-NSC collections and from nature biotopes as well. Such integrative reference genome construction will provide an important resource both for advanced molecular breeding and fundamental researches in plant biology and ecology based on genomic approaches using $\boldsymbol{F}$. carica and $\boldsymbol{H}$. ligusticifolium as model species.
\end{abstract}

Keywords - NGS technologies, hybrid genome assembly, Ficus carica, Heracleum ligusticifolium

\section{Introduction}

Modern next-generation sequencing technologies (NGS) provide new opportunities for plant genomics research and allow to advance in the field of biotechnology and molecular breeding. Despite a wide range of the targets, methodologies and tools, high quality genome assembly is a key requirement for a successful implementation of the projects associated with whole-genome studies [1]. Additionally, some issues, for example in the field of molecular breeding, require obtaining the most complete information about intraspecies genetic diversity (the so-called "pangenom") or the genomic architecture of the genus ("superpangenom"). The number of plants with a well annotated genome is currently limited to a few dozen of model and / or economically valuable species which significantly limits the use of advanced genomic tools for genotyping, targeted search of quantitative trait locus (QTL) and realization of molecular breeding methodology in general [2]. Development of high-throughput NGS technologies was accompanied by a constant decline of analysis cost. And now they are already affordable for comprehensive scientific researches, so the global screening of plant genomic diversity is on the agenda.

The Nikita Botanical Gardens (NBG) is one of the oldest scientific and breeding centers in Russia with thousands of species and cultivars of plants that are grown as ex situ as in vitro conditions. The NBG plant collection incorporates extensive gene pool of garden, essential-oil, ornamental and other valuable crops, as well as wild plants, including rare and endemic species. Scientific researches based on these collections have been ongoing for more than 150 years. Various biotechnological methods of plant propagation and conservation have been used for the past three decades. Regular molecular-genetics investigations were started a few years ago and several projects are underway. Genotyping of the valuable crops, phytopathogens analyzing, development of new breeding approaches with searching for QTL and marker selection consist the main applied purposes. In addition, studies in the field of functional genomics are started. Entire transcriptomes for several plant species were sequenced to investigate the molecular mechanisms of plant adaptation to various stress factors, as well as cell regulation of somatic embryogenesis and regeneration based on analysis of differential gene expression. All these studies are integrated in the framework of scientific program of genomic screening of the NBG-NSC plant collections targeted at comprehensive description of their molecular diversity in close connection with morphological, physiological and others phenotype features. Strategic goal of the program is to develop and implement advanced molecular approaches for plant biotechnology, breeding, propagation and conservation routine works. Creation of high-quality reference genomes is the key stage for the subsequent successful implementation of the project aims.

\section{Modeling plant species}

Two whole-genome sequencing projects to produce high quality assembly of the reference genomes of the common fig Ficus carica L. (Moraceae) and Crimean endemic species Heracleum ligusticifolium M. Bieb. (Apiaceae) have started. Common fig is one of the most important subtropical fruits used in food, cosmetic, and soap industries as well as in medicine. The NBG has a large $F$. carica collection which includes 267 cultivars and hybrid forms. A special research program aimed at a comprehensive description of the genetic diversity of the common fig collection, including sequencing of the key cultivars, genotyping of the collection, QTL analysis of the valuable horticultural traits are forming. 
Heracleum is the largest genus of the Apiaceae family with a broad spectrum of phytochemistry and biological activities but without any whole-genome sequencing data. $H$. ligusticifolium possesses a number of unique adaptive properties, including those associated with somatic embryogenesis, which ensure the successful survival of this endemic species within a highly restricted distribution area under the extremely adverse environmental conditions. To date assembling of the high-quality reference Heracleum genome is the most critical step to advance molecular researches not only for the Crimean endemic $H$. ligusticifolium but also for the genus as whole. Deciphering the biochemical pathways caused by stress and the targeted search for specific genes involved in adaptive reactions are the main tasks for the future investigation after the creation and subsequent annotation of the H. ligusticifolium genome.

\section{Results}

Leaves of microshoots in $F$. carica cultivar 'Sabrutsiya Rozovaya' and in H. ligusticifolium cultured in vitro were used to high molecular weight DNA isolation using GeneJET Genomic DNA Kit by standard protocol. The entire genomes have been sequenced using Oxford Nanopore MinION and Illumina NextSeq550 instruments as well. The genomic data were preliminary assembled in Canu v.1.8 software package. Oxford Nanopore technology allows to get extremely long reads, but such readings are known for having too high error rates. Whereas accurate short-reads sequencing by Ilumina does not allow to resolve genome regions with high repeated and duplicated sequences, especially in the complex and polyploid plant genomes. A hybrid assembly using combined data sets with long and short reads is a promising approach to create an accurate and well-resolved reference genome [3]. Several workflows including pipelines for a haplotyperesolved de novo genome assembly have been tested.
Assessment of assembly genome quality in terms of contiguity, accuracy, and completeness was done. As a result, the first versions of the reference genomes of $F$. carica cultivar 'Sabrutsiya Rozovaya' and H. ligusticifolium were made, and their annotations are performing. Subsequent improvement and development of the primary genome assemblies up to "pan-genome" rank will be carried out using advanced bioinformation approaches and computational tools by assimilation of new sequenced genomes and transcriptomes of the model species taken from the NBS-NSC collections and from nature biotopes as well. Such integrative reference genome construction will provide an important resource both for advanced molecular breeding and fundamental researches in plant biology and ecology based on genomic approaches [4] using $F$. carica and $H$. ligusticifolium in our laboratory as model species.

\section{Acknowledgment}

The studies are done at the Kurchatov Genomic Center NBG-NSC of the RAS and supported by the grant № 075-152019-1670 of the Ministry of Science and Higher Education of the Russian Federation.

\section{References}

[1] Bailey-Serres J. et al (2019) Genetic strategies for improving crop yields. Nature. 575(7781): 109-118. doi:10.1038/s41586-019-1679-0.

[2] Yuan Y. et al. (2017) Improvements in Genomic Technologies: Application to Crop Genomics. Trends in Biotechnology. 35 (6): 547 -558 .

[3] Jung H. et al. (2019) Tools and Strategies for Long-Read Sequencing and De Novo Assembly of Plant Genomes. Trends in Plant Science. doi:10.1016/j.tplants.2019.05.003

[4] Usai G. et al. (2020) Epigenetic patterns within the haplotype phased fig (Ficus carica L.) genome. Plant J. doi:10.1111/tpj.14635 